\title{
Radiologic Findings of Metastatic Malignant Melanoma of the Breast: Mammographic, Sonographic, Dynamic Contrast-Enhanced Breast MRI, and 18F-FDG PET-CT Features
}

\author{
Youyeon Kim, ${ }^{1}$ Kyu Ran Cho, ${ }^{1,}$ Bo Kyoung Seo, ${ }^{2}$ Ok Hee Woo, ${ }^{3}$ Jeong Hyun Lee, ${ }^{4}$ and Sung Bum Cho ${ }^{1}$ \\ ${ }^{1}$ Department of Radiology, Korea University Anam Hospital, Seoul, Korea \\ ${ }^{2}$ Department of Radiology, Korea University Ansan Hospital, Ansan, Korea \\ ${ }^{3}$ Department of Radiology, Korea University Guro Hospital, Seoul, Korea \\ ${ }^{4}$ Department of Pathology, Korea University Anam Hospital, Seoul, Korea \\ "Corresponding author: Kyu Ran Cho, Department of Radiology, Korea University Anam Hospital, Seoul, Korea. Tel: + 82-29205657, Fax: + 82-29293796, E-mail: krcho@korea.ac.kr
}

Received 2016 May 13; Revised 2016 August 03; Accepted 2016 September 10.

\begin{abstract}
Metastatic involvement of the breast from an extra-mammary tumor is rare. However, when a patient with a known primary malignancy complains of a palpable breast mass, both the primary breast cancer and the metastatic lesion must be considered as a differential diagnosis. Melanoma is one of the tumors that commonly metastasizes to the breast, but the characteristic imaging features are not well known. Therefore, an understanding of the characteristic image findings for melanoma would be advantageous in differentiating a breast mass in patients with a history of melanoma. A 73-year-old woman with a history of recurring melanoma in the nasal cavity presented with a palpable mass in the left breast. She had undergone various imaging studies, which revealed two masses in the left breast and an enlarged right intramammary lymph node. The patient underwent lumpectomy of masses in the left breast for tissue confirmation, and pathologic examination revealed metastatic melanoma. Melanoma exhibits typical signal intensity on MRI and commonly metastasizes to the breast. Therefore, it is important to be aware of the characteristic imaging findings for metastatic melanoma, particularly when a melanoma patient presents with a breast lump.
\end{abstract}

Keywords: Breast Neoplasm, Metastasis, Melanoma, Mammography, Magnetic Resonance Imaging (MRI)

\section{Introduction}

Melanoma arises from melanocytes in the basal layer of the epidermis and may originate on the trunk, extremities, face, eye, mucosal surfaces, and rarely, other visceral organs (1-3). Metastases are common in the subcutaneous and soft-tissue locations, and MRI has an important role in evaluating brain, bone marrow, liver, and softtissue metastases (3-5). Melanoma commonly metastasizes to the breast, but metastatic involvement of the breast parenchyma is rare, accounting for $0.5-6.6 \%$ of all breast malignancies (6-9). There are few reports on the imaging findings of metastatic involvement of the breast in malignant melanoma because the incidence is extremely low. Some studies have described mammography and sonography findings, but little is known about the MRI findings of melanoma metastasis to the breast parenchyma. Here, we report a case of breast parenchymal metastasis of malignant melanoma and describe the findings of mammography, sonography, CT, MRI, and 18F-fluorodeoxyglucose positron emission tomography -computed tomography (18F-FDG PET-CT).

\section{Case Presentation}

A 73-year-old woman presented with a palpable mass and pain in the left breast for 5 months. She had a history of malignant melanoma. The first diagnosis was made in 1997 by a right total inferior turbinectomy for a nasal cavity mass. She underwent several additional procedures for the wide excision of the right nasal cavity and a right medial maxillectomy with consecutive radiation therapy for recurrent melanoma over the next 15 years. In 2013, she complained of right periorbital swelling and diplopia, which were found to be caused by a protruding mass with black spots in the right lateral wall of the nasal cavity. This mass was recurrent melanoma. Craniofacial resection was performed for treatment. In 2015, the diagnosis of recurrent melanoma was confirmed for a newly developed nasal cavity mass. By this time, all recurrences were found to be present in the head and on the ipsilateral side, although she complained of symptoms in the breast on the contralateral side.

The patient had no family history of breast cancer. There was no skin retraction, nipple discharge, or palpable axillary lymph nodes on physical examination. On mammography (Hologic Inc., Bedford, MA, USA) that is shown 
in (Figure 1A), two circumscribed round to oval shaped hyperdense and isodense masses were seen in the left mid to inner deep portion. The maximal diameter of the hyperdense mass was $5 \mathrm{~cm}$, and that of the isodense mass was $1.2 \mathrm{~cm}$. Microcalcifications and enlarged lymph nodes were not detected. She had brought sonographic images acquired at a different hospital. An approximately $5-\mathrm{cm}$ circumscribed hypoechoic mass was seen on sonography. Breast MRI (Figure 1B - D) was performed using a 3.0-T Scanner (Achieva 3.0T TX; Philips Healthcare, Best, the Netherlands) with a breast coil (MRI Devices; InVivo Research, Orlando, FL, USA) with the patient in the prone position. Images were acquired in the axial plane with the following sequences: axial, diffusion-weighted, spin-echo singleshot echo-planar-imaging, with diffusion-sensitizing gradients (TR/TE, 5471/72; b values, 0,600 and $1000 \mathrm{~s} / \mathrm{mm}^{2}$; image matrix, $96 \times 126$; field of view [FOV], $320 \times 320$ $\mathrm{mm}$; section thickness, $3 \mathrm{~mm}$; section gap, $0 \mathrm{~mm}$; three signal acquired; acquisition time, 80 seconds); axial, T2weighted, fat-suppressed, fast spin-echo imaging (TR/TE, 5727/70; flip angle, $90^{\circ}$; field of view [FOV], $581 \times 342 \mathrm{~mm}$; image matrix, $620 \times 309$; section thickness, $3 \mathrm{~mm}$ section gap, $0 \mathrm{~mm}$ ); pre- and post-contrast enhanced, axial, T1-weighted three-dimensional (3D) fast spoiled gradient echo sequence (TR/TE, 6/3; flip angle, $0^{\circ}$; FOV, 330 × 340 mm; image matrix, $436 \mathrm{x} 436$; section thickness, $3 \mathrm{~mm}$ section gap, $1.5 \mathrm{~mm}$ ). A volume of $15 \mathrm{~mL}$ of Gadodiamide (Omniscan, GE Healthcare, Oslo, Norway) was injected intravenously into the antecubital vein with a power injector (Spectris; Medrad, Indianola, PA, USA) at a rate of $2 \mathrm{~mL} / \mathrm{s}$. Imaging was performed before the injection and six times after the injection: immediately and then at intervals of 1 minute. The image post-processing included subtracting the unenhanced images from the enhanced images and generating 3D maximum-intensity projections using the first contrast-enhanced series. The interpretation of the degree and pattern of the enhancement was performed using computer-aided detection (CAD) stream TM (Merge health care, Chicago, IL, USA). A circumscribed round-shaped enhancing mass was seen in the left upper inner quadrant. The center of the mass was in the breast parenchyma, and the mass was broadly attached to the underlying pectoralis muscle. There was no visible fat plane between the mass and the muscle, which indicates the possibility of pectoralis invasion. A small-circumscribed round-shaped mass was seen at the lower outer aspect of the mass. These two masses showed high signal intensity on both T1- and fat-saturated T2-weighted images, and they showed early fast enhancement with a delayed wash-out pattern on dynamic study. All images showed diffusion restriction. Because the signals and enhancement patterns were similar, these two masses could be considered to be of the same na- ture. An enlarged enhancing lymph node was noted in the right internal mammary area. There was no significantly enlarged lymph node in either axillae. Additionally, a chest CT scan was performed (Figure 1E), in which the larger mass showed a central hypodense area that might indicate associated necrosis. Loss of the fat plane between the mass and the pectoralis muscle was also observed. There was no pathologic finding in either lung.

Before any surgical treatment, she had undergone 18FFDG PET-CT (Figure 1F) to detect other metastatic lesions throughout the body. Two masses in the left breast and the enlarged right internal mammary lymph node showed hypermetabolism. The maximal standard uptake value (SUV) for the larger mass in the left breast was 7.2. There were no other hypermetabolic lesions in the other parts of the body beside the known recurred mass in the right nasal cavity.

The patient underwent a lumpectomy for the palpable and painful mass in the left breast (Figure 2). Histopathologic examinations revealed cells with vesicular nuclei and prominent nucleoli, which indicate the lack of full maturation. In addition, there were dense infiltrations of melanophages.

\section{Discussion}

Although primary breast cancer is one of the most common malignancies in women, the breast is an unusual site for metastasis from extramammary tumors (10, 11). According to previous reports, the incidence of nonprimary tumors of the breast ranges from $0.5 \%$ to $6.6 \%$ of all breast malignancies $(6,7,12)$. Variable origins of metastases to the breast have been reported in previous studies $(9,13)$. In declining order, the following tumors are the most common sources: lymphoma, melanoma, rhabdomyosarcoma, lung cancer, ovarian cancer, gastrointestinal, and genitourinary tumors $(9,13,14)$. It is well known that the presence of breast metastases is a poor prognostic factor $(6,15)$.

Metastasis of melanoma to the breast tissue usually occurs through hematogeneous spread. Due to the good vascularity and the presence of more glandular tissue in the outer portion of the breast, metastasis is more common in the outer portion $(12,16,17)$. Most studies have reported that a solitary lesion is more common than multiple lesions or bilateral involvement $(6,12,15,16)$. In the present case, the patient complained of a palpable mass in the left breast, which was found to be two distinct masses. The masses were located in the upper inner to middle portion, rather than the outer portion.

There are several reports of melanoma metastases to the breast, but there are few studies reporting the imaging findings. Most metastatic lesions to the breast look 

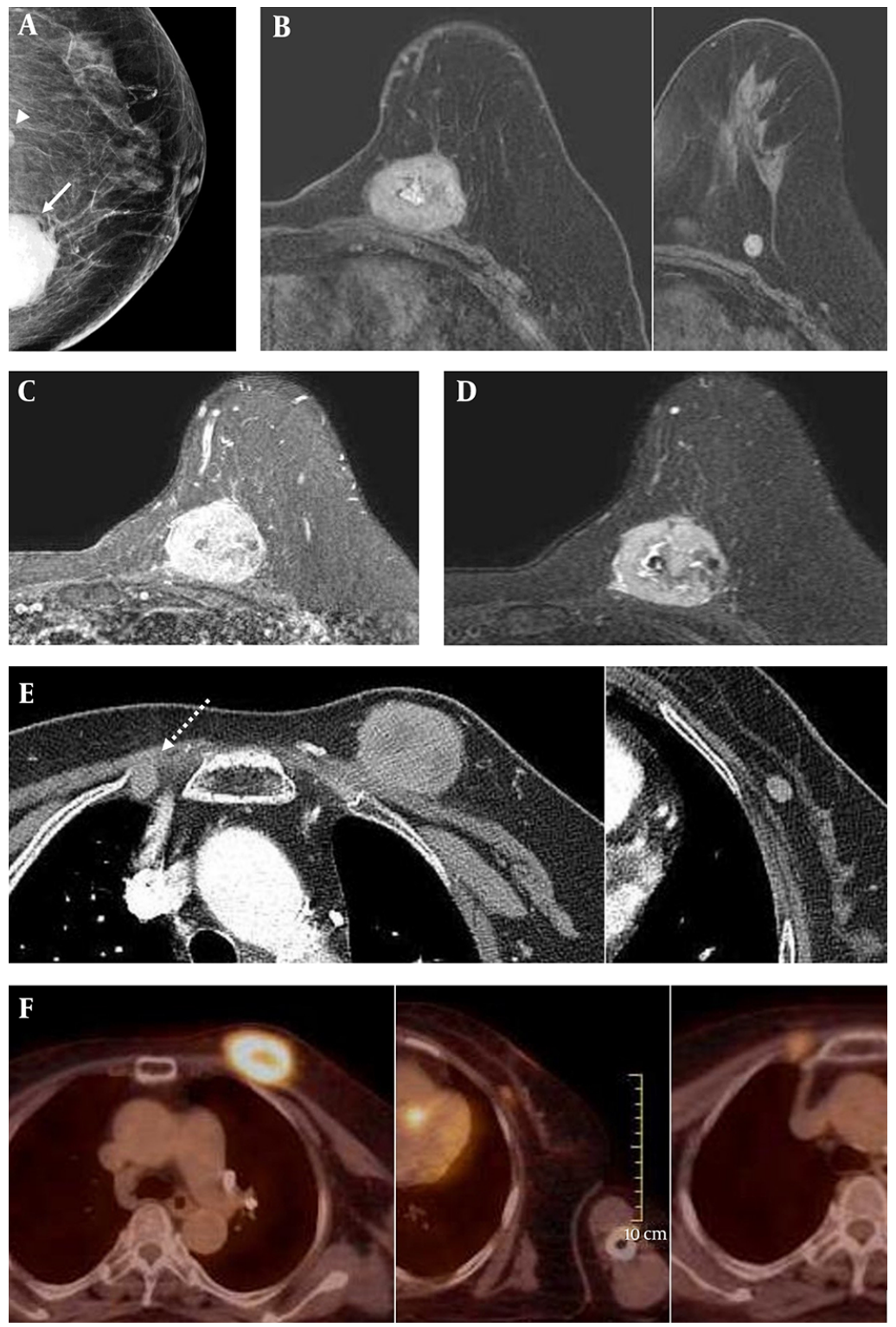

Figure 1. A 73-year-old woman with a palpable mass and pain in the left breast and a history of malignant melanoma. On mammography showing the left craniocaudal (CC) view (A), two circumscribed round to oval shaped hyperdense (solid arrow) and isodense (arrowhead) masses are seen in the left mid to inner deep portion, where the patient complained of a palpable mass. On sonography taken at a different hospital (not shown), the masses were circumscribed and had low echogenicity. The masses all showed mostly high signal intensity on axial fat-saturated T1 weighted imaging (B) and showed enhancement on contrast-enhanced T1 weighted image (C). They also showed high signal intensity on fat-saturated T2 weighted image (D). On chest CT scan (E), the larger mass showed a central hypodense area of possible necrosis. A loss of the fat plane in between the larger mass and the pectoralis muscle is also seen, indicating the possibility of muscle involvement. Additionally, an enlarged internal mammary lymph node on the contralateral side is noted (dashed arrow). On 18F-fluorodeoxyglucose positron emission tomography -computed tomography (18F-FDG PET-CT) (F), the masses and enlarged internal mammary lymph node all had increased FDG uptake, and the maximum SUV of the large mass was 7.2.

similar to those of primary breast cancer. However, according to previous studies, the most common mammographic findings of metastatic lesions are single or multiple circumscribed round masses of variable size that may have slightly irregular margins $(9-11,13,14)$. On sonography, metastatic lesions of previously reported studies ex- hibited various morphologies: circumscribed or irregular margins, hyperechoic or hypoechoic, with or without posterior acoustic shadowing, and round to oval shape (9-11, 13). The lesions are usually not associated with spiculation, microcalcification, or secondary skin or nipple change, which indicate no associated desmoplastic response in 

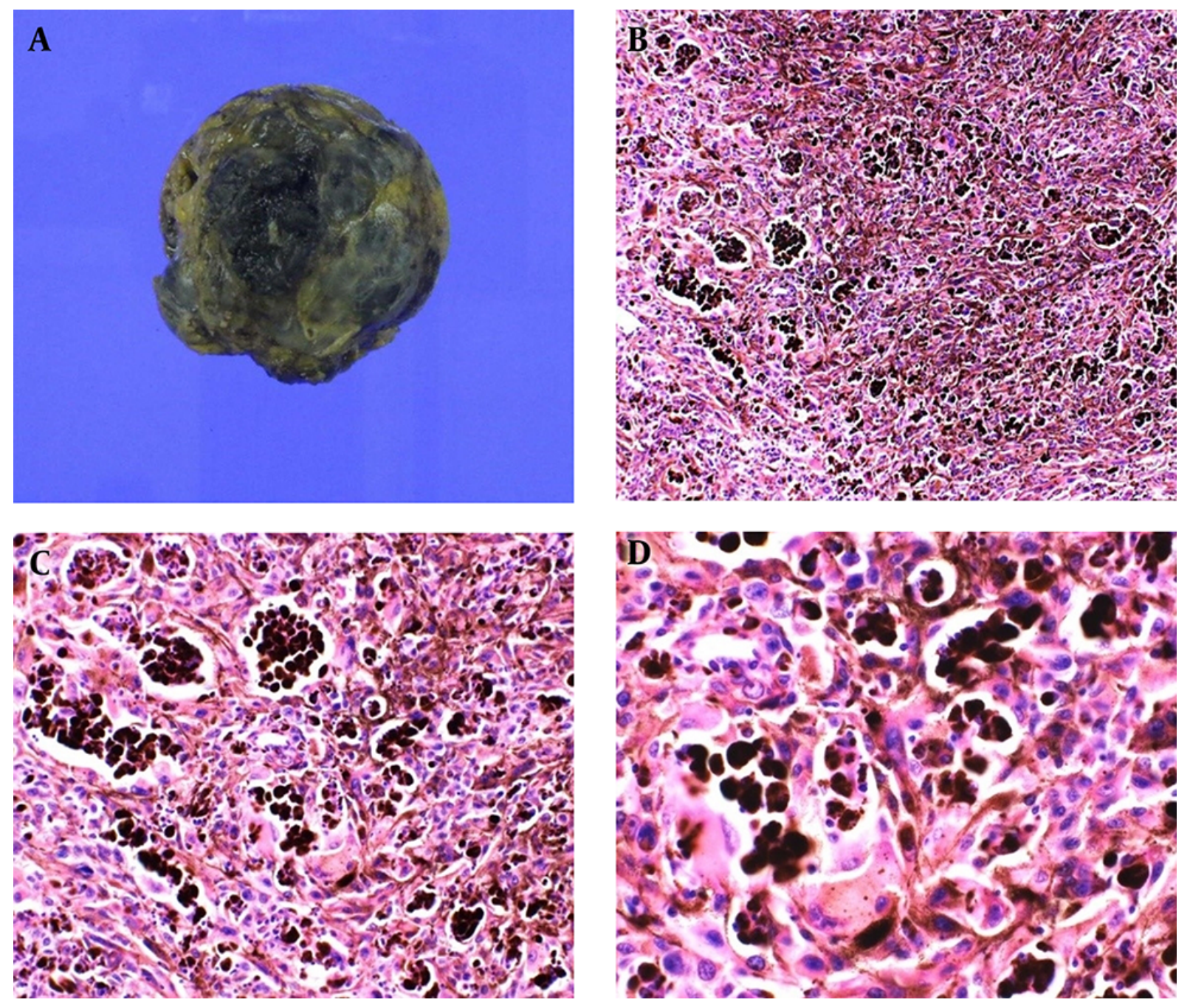

Figure 2. Cut surface (A) of the larger mass shows diffusely dark brownish color and a hemorrhagic and necrotic appearance. On microscopic examination (hematoxylineosin stain, original magnification $\times 100$ (B), $\times 200$ (C), $\times 400$ (D)), dense infiltrations of melanophages are noted. Additionally, cells with vesicular nuclei and prominent nucleoli are seen, indicating lack of full maturation.

contrast to the primary breast cancer $(9-11,13)$. In our case, there were two masses in the left upper mid to inner breast. Both masses had circumscribed margins and were round in shape on mammography. These masses did not have any associated findings such as spiculation, microcalcifications, or skin or nipple changes. Unfortunately, only scanned images of sonographs taken at another hospital were available. Those sonographic images showed a circumscribed hypoechoic mass. All of those findings were consistent with previously reported image findings of metastatic lesions in the breast. This indicates that metastatic melanoma looks similar to other metastatic lesions in the breast on mammography and sonography.

Magnetic resonance imaging is useful in evaluating lesions in patients with dense breast parenchyma (11). Metastatic lesions to the breasts detected on MRI appear similar using other imaging modalities. Such lesions may be single or multiple and circumscribed and round in shape without signs of desmoplastic reaction $(8,11,13,14$, 17). As melanoma contains a melanin component that contains stable free paramagnetic radicals that have a paramagnetic effect and result in a shortening of the T1 and T2 relaxation times (8), melanoma possesses a high signal on T1weighted images and a low signal on T2-weighted images on $\operatorname{MRI}(8,17,18)$. Thus, when a tumor in the breast shows this typical signal intensity on MRI, radiologists must consider melanin-containing tumors, and they should investigate the patient's clinical history and perform a biopsy 
for tissue confirmation. On diffusion-weighted imaging, melanoma generally shows diffusion restriction and will enhance after intravenous (IV) gadolinium-based contrast agent administration (3). This typical signal intensity of melanoma can be lost when it is associated with the subacute to chronic stage of hematoma. When the tumor is associated with hemorrhage and transition from subacute to chronic stage, it can be seen as a hyperintense mass on both T1- and T2-weighted images. In the present case, the masses were seen as hyperintense on both T1and T2-weighted images. Diffusion restriction was evident on diffusion-weighted imaging, and enhancement was observed on contrast-enhanced T1-weighted imaging. The mass did not follow the typical signal intensity of melanoma on the T2-weighted images in that it exhibited a high T2 signal intensity rather than typical low T2 signal of melanoma. The hematoma associated with the mass could be one of the reasons of the atypical signal, because the patient in this case underwent sonography-guided biopsy of the mass to obtain tissue confirmation approximately one week before taking MRI. However, this hypothesis cannot be applied to the other small mass that did not experience biopsy. Since melanoma is known to be frequently associated with internal hemorrhage, spontaneous internal hemorrhage might be one possible reason of atypical signal intensity.

According to the national comprehensive cancer network guidelines for melanoma imaging workup, contrastenhanced CT or PET-CT is recommended for stage IV melanoma. CT scan can be used to determine the presence of enlarged lymph nodes, swelling of the breast tissue, and involvement of the chest wall with loss of the tissue plane (10). In this case, we could see involvement of the pectoralis muscle by detecting loss of the fat plane in between the mass and the muscle on a CT scan. On PET-CT, a lesion with a maximum SUV of more than 1.5 is considered to have an increased FDG uptake. Previous studies have reported that melanomas are FDG avid on PET-CT $(3,19,20)$. We could also identify masses with increased FDG uptake, and the maximum SUV was 7.2.

There are only a few radiologic reports of metastatic melanoma in the breast tissue and none of them described MRI findings in detail. Melanoma exhibits the typical signal intensity on MRI, and considering that melanoma is one of the most common primary tumors that metastasize to the breast tissue, it is important to know the characteristic imaging findings of metastatic melanoma. Even though metastasis to the breast is very rare, one should consider metastatic melanoma when a patient with a known history presents with a palpable mass in the breast that shows characteristic imaging findings. A careful assessment and tissue confirmation for the diagnosis should be recommended for development of the treatment plan.

\section{Footnotes}

Authors' Contributions: Study concept and design: Youyeon Kim, and Kyu Ran Cho; Acquisition of data: Youyeon Kim, and Kyu Ran Cho; Analysis and interpretation of data: Youyeon Kim, Kyu Ran Cho, and Jeong Hyun Lee; Drafting of the manuscript: Youyeon Kim; Critical revision of the manuscript for important intellectual content: Kyu Ran Cho, Bo Kyoung Seo, and Ok Hee Woo; Administrative, technical, and material support: Bo Kyoung Seo, and Sung Bum Cho; Study supervision: Kyu Ran Cho, and Sung Bum Cho

Financial Disclosure: This report did not receive commercial financial support and the authors have no conflicts of interest.

Funding/Support: This case report did not receive commercial financial support.

\section{References}

1. Patnana M, Bronstein Y, Szklaruk J, Bedi DG, Hwu WJ, Gershenwald JE, et al. Multimethod imaging, staging, and spectrum of manifestations of metastatic melanoma. Clin Radiol. 2011;66(3):224-36. doi: 10.1016/j.crad.2010.10.014. [PubMed: 21295201].

2. Katalinic A, Kunze U, Schafer T. Epidemiology of cutaneous melanoma and non-melanoma skin cancer in Schleswig-Holstein, Germany: incidence, clinical subtypes, tumour stages and localization (epidemiology of skin cancer). BrJ Dermatol. 2003;149(6):1200-6. [PubMed: 14674897].

3. Juan YH, Saboo SS, Tirumani SH, Khandelwal A, Shinagare AB, Ramaiya N, et al. Malignant skin and subcutaneous neoplasms in adults: multimodality imaging with CT, MRI, and 18F-FDG PET/CT. AJR Am J Roentgenol. 2014;202(5):422-38. doi: 10.2214/AJR.13.11424. [PubMed: 24758677].

4. Lee CC, Faries MB, Wanek LA, Morton DL. Improved survival for stage IV melanoma from an unknown primary site. J Clin Oncol. 2009;27(21):3489-95. doi: 10.1200/JCO.2008.18.9845. [PubMed: 19451446].

5. King DM. Imaging of metastatic melanoma. Cancer Imaging. 2006;6:204-8. doi: 10.1102/1470-7330.2006.0033. [PubMed: 17208677].

6. Amichetti M, Perani B, Boi S. Metastases to the breast from extramammary malignancies. Oncology. 1990;47(3):257-60. [PubMed: 2342767].

7. Bohman LG, Bassett LW, Gold RH, Voet R. Breast metastases from extramammary malignancies. Radiology. 1982;144(2):309-12. doi: 10.1148/radiology.144.2.7089284. [PubMed: 7089284].

8. Ho LW, Wong KP, Chan JH, Chow LW, Leung EY, Leong L. MR appearance of metastatic melanotic melanoma in the breast. Clin Radiol. 2000;55(7):572-3. doi: 10.1053/crad.1999.0102. [PubMed: 10924384].

9. Vizcaino I, Torregrosa A, Higueras V, Morote V, Cremades A, Torres $\mathrm{V}$, et al. Metastasis to the breast from extramammary malignancies: a report of four cases and a review of literature. Eur Radiol. 2001;11(9):1659-65. doi: 10.1007/s003300000807. [PubMed: 11511887].

10. Yang WT, Muttarak M, Ho LW. Nonmammary malignancies of the breast: ultrasound, CT, and MRI. Semin Ultrasound CT MR. 2000;21(5):375-94. [PubMed: 11071618]. 
11. Bartella L, Kaye J, Perry NM, Malhotra A, Evans D, Ryan D, et al. Metastases to the breast revisited: radiological-histopathological correlation. Clin Radiol. 2003;58(7):524-31. [PubMed: 12834635].

12. Toombs BD, Kalisher L. Metastatic disease to the breast: clinical, pathologic, and radiographic features. AJR Am J Roentgenol. 1977;129(4):673-6. doi:10.2214/ajr.129.4.673. [PubMed: 409241].

13. Akcay MN. Metastatic disease in the breast. Breast. 2002;11(6):526-8. doi: 10.1054/brst.2002.0467. [PubMed: 14965721].

14. Chung SY, Oh KK. Imaging findings of metastatic disease to the breast. Yonsei Med J. 2001;42(5):497-502. doi: 10.3349/ymj.2001.42.5.497. [PubMed: 11675677].

15. McCrea ES, Johnston C, Haney PJ. Metastases to the breast. AJR Am J Roentgenol. 1983;141(4):685-90. doi: 10.2214/ajr.141.4.685. [PubMed: 6604418].

16. Vergier B, Trojani M, de Mascarel I, Coindre JM, Le Treut A. Metastases to the breast: differential diagnosis from primary breast carcinoma. J Surg Oncol. 1991;48(2):112-6. [PubMed:1921396].
17. Al Samaraee A, Khout H, Barakat T, Fasih T. Breast metastasis from a melanoma. OchsnerJ. 2012;12(2):149-51. [PubMed: 22778680].

18. Premkumar A, Marincola F, Taubenberger J, Chow C, Venzon D, Schwartzentruber D. Metastatic melanoma: correlation of MRI characteristics and histopathology. J Magn Reson Imaging. 1996;6(1):190-4 [PubMed: 8851427].

19. Strobel K, Dummer R, Husarik DB, Perez Lago M, Hany TF, Steinert HC. High-risk melanoma: accuracy of FDG PET/CT with added CT morphologic information for detection of metastases. Radiol ogy. 2007;244(2):566-74. doi: 10.1148/radiol.2442061099. [PubMed: 17641374].

20. Bronstein Y, Ng CS, Rohren E, Ross MI, Lee JE, Cormier J, et al. PET/CT in the management of patients with stage IIIC and IV metastatic melanoma considered candidates for surgery: evaluation of the additive value after conventional imaging. AJR Am J Roentgenol. 2012;198(4):902-8. doi: 10.2214/AJR.11.7280. [PubMed: 22451559]. 